\title{
Controlling Boron Diffusion during Rapid Thermal Annealing with Co-Implantation by Amphoteric Impurity Atoms
}

\author{
Yu. V. Makarevich ${ }^{a}$, F. F. Komarov ${ }^{a}$, A. F. Komarov ${ }^{a}$, A. M. Mironov ${ }^{a}$, \\ G. M. Zayats ${ }^{b}$, and S. A. Miskevich ${ }^{a}$ \\ ${ }^{a}$ Sevchenko Institute of Applied Physics Problems, Minsk, 220108 Belarus \\ ${ }^{b}$ Institute of Mathematics, National Academy of Sciences, Minsk, 220072 Belarus \\ e-mail:elionics@bsu.by,zayats@im.bas-net.by
}

\begin{abstract}
A model for simulating the rapid thermal annealing of silicon structures implanted with boron and carbon is developed. The model provides a fair approximation of the process of boron diffusion in silicon, allowing for such effects as the electric field, the impact of the implanted carbon, and the clustering of boron. The migration process of interstitials is described according to their drift in the field of internal elastic stress.
\end{abstract}

DOI: $10.3103 / \mathrm{S} 1062873812050164$

\section{INTRODUCTION}

Low-depth doping by impurities must be performed to create submicrometer semiconductor devices and integrated VLSI microchips. The main difficulties are in this case related to the formation of shallow regions with $p$-type conductivity. The boron atoms predominantly used in creating $p$-regions in silicon have high diffusivity. In addition, the effect of nonequilibrium accelerated atomic diffusion of boron in silicon is observed upon post-implantation thermal annealing. Rapid thermal annealing is one solution to the problem, but it does not allow us to compensate for this effect entirely.

To suppress the effect of nonequilibrium accelerated atomic diffusion of boron in silicon, we can use implantation by carbon atoms - junctions created by the combined implantation of boron and carbon in silicon have a much shallower localization depth than those formed without carbon implantation [1].

The aim of this work is to construct a diffusion model of boron implantation in silicon with allowance for the implanted carbon atoms. Boron diffusion is examined simultaneously with the effect of migrating nonequilibrium defects. The impact of elastic stress on the behavior of point defects is also considered.

\section{DESCRIPTION OF THE MODEL}

We assume that the boron diffusion in silicon is caused by the formation, migration and dissociation of mobile pairs created by impurity atoms, interstitial atoms, and by the influence of an electric field [2-4]. We also assume that boron diffusion is insignificantly affected by such vacancies, and the carbon distribution is stationary during the period of thermodiffusion.
The equation for the diffusion of boron atoms is in this case

$$
\begin{gathered}
\frac{\partial C^{T}}{\partial t}=-\frac{\partial J}{\partial x} \\
=\frac{\partial}{\partial x}\left(D(\chi)\left(\frac{\partial\left(C^{I} C\right)}{\partial x}+\frac{C^{I} C}{\sqrt{(C-N)^{2}+4 n_{e}^{2}}} \frac{\partial C}{\partial x}\right)\right),
\end{gathered}
$$

where $C$ is the active concentration of boron; $J$ is boron flux; $N$ is the donor impurity concentration; $C^{T}$ is the total boron concentration; $C^{I}$ is the defect concentration; $n_{e}$ is the concentration of intrinsic carriers; $D(\chi)=D_{i} \frac{1+\beta_{1} \chi+\beta_{2} \chi^{2}}{1+\beta_{1}+\beta_{2}}$ is the effective diffusion coefficient of impurity atoms in the field of elastic stress via the mechanism of formation of the complexes with intrinsic interstitial atoms; $\chi=n / n_{e}$ is the normalized concentration of charge carriers; $D_{i}$ is the diffusion coefficient of boron; and $\beta_{1}$ and $\beta_{2}$ are coefficients that determine the relative contribution from singly and doubly charged defects in the process of boron diffusion.

Let us define the boundary conditions for Eq. (1). Flux $J$ on the surface of the crystal is assumed to be

$$
J=K_{s} C+\mu,
$$

where $K_{s}$ is the rate of impurity transport through the surface, and $\mu$ is a parameter characterizing the constant component of the rate of the impurity evaporation. On the right boundary of the range of modeling (in the bulk of the substrate), we set $J=0$.

The initial conditions have the form

$$
\left.C^{T}(x, t)\right|_{t=0}=C_{0}(x),
$$


where $C_{0}(x)$ is the implanted profile of the impurity. Allowing for the influence of the implanted interstitial carbon atoms, the nonstationary equation for the defects takes the form

$$
\begin{aligned}
\frac{\partial C^{I}}{\partial t} & =\frac{\partial}{\partial x}\left(d(\chi) \frac{\partial C^{I}}{\partial x}\right)-\frac{\partial}{\partial x}\left(v C^{I}\right) \\
& -\frac{\left(1+k C^{c}\right) C^{I}}{\left(l_{i}\right)^{2}}+\frac{d(\chi)}{\left(l_{i}\right)^{2}}
\end{aligned}
$$

where $d(\chi)$ is the effective diffusion coefficient of the interstitial silicon atoms, $C^{c}$ is the carbon concentration, $k$ is a parameter, and $l_{i}$ and $v$ are the migration length and the drift velocity of the point defects.

To describe the drift velocity in the field of elastic stresses, we use the equation [5]

$$
v=v_{x}=-d^{C}(\chi) \frac{1}{k_{B} T} \frac{\partial U^{d}}{\partial x},
$$

where $d^{C}(\chi)$ is the cumulative effective diffusion coefficient of the interstitial defects, $k_{B}$ is the Boltzmann constant, and $U^{d}$ is the potential energy of point defects in the field of elastic stresses. In our model, we considered the simplest approximation for the effective drift coefficient of interstitials:

$$
d^{C}(\chi)=\text { const. }
$$

Assuming that the potential energy in the field of elastic stresses is proportional to the carbon concentration, the resulting value for the drift velocity is given by

where $B$ is a constant.

$$
v_{x}=-\frac{B}{k_{B} T} \frac{\partial C^{c}}{\partial x}
$$

We assume that in the bulk of semiconductor, the total defect concentration is equal to the concentration of intrinsic defects, while on the surface we have the condition

$$
d(\chi) \frac{\partial C^{I}}{\partial x}=\beta C^{I}+\gamma,
$$

where $\beta$ is a recombination coefficient, and $\gamma$ is the surface length of recombination. The initial conditions are determined by the profiles of implantation.

\section{NUMERICAL ALGORITHM}

An approximate solution to the problem is found using the finite difference method [6]. The following temporal and spatial meshes were used:

$$
\begin{aligned}
& \omega_{\tau}=\left\{t_{j}=j \tau, \quad j=0, \quad 1, \ldots, j_{0}, \quad j_{0} \tau=t_{f}\right\}, \\
& \omega_{h}=\left\{x_{i}=i h, \quad i=0,1, \ldots, N, \quad N h=l\right\} .
\end{aligned}
$$

We take the designations for finite difference relationships analogous to those used in [6]. Let $y, \tilde{y}, z$ be the approximate values for $C, C^{I}$ and $\chi$, respectively.
Using the mesh $\omega_{h \tau}=\omega_{h} \times \omega_{\tau}$, the conservative advanced finite-difference scheme for Eq. (1) is given by

$$
\begin{gathered}
\left.y_{\bar{t}}\right|_{i} ^{j}=\left.\left(a_{1}(z)(y \tilde{y})_{\bar{x}}\right)_{x}\right|_{i} ^{j}+\left.\left(a_{2}(z, y, \tilde{y}) y_{\bar{x}}\right)_{x}\right|_{i} ^{j}, \\
i=1,2, \ldots, N-1, j=1,2, \ldots, j_{0} . \\
a_{1}(z)=0.5\left(D\left(z_{i}\right)+D\left(z_{i-1}\right)\right), \\
a_{2}(z, y, \tilde{y})=0.5\left(\frac{D\left(z_{i}\right) \tilde{y}_{i} y_{i}}{\sqrt{y_{i}-N+4 n_{e}^{2}}}+\frac{D\left(z_{i-1}\right) \tilde{y}_{i-1} y_{i-1}}{\sqrt{y_{i-1}-N+4 n_{e}^{2}}}\right) .
\end{gathered}
$$

The difference condition at $x=0$ has the form

$$
\begin{gathered}
\left.\left.0.5 h y_{\bar{t}}\right|_{i=0} ^{j}=\left.a_{1}(z)(\tilde{y} y)_{\bar{x}}\right|_{i=1} ^{j}+a_{2}(z, y, \tilde{y}) y_{\bar{x}}\right)\left.\right|_{i=1} ^{j}-\left.K_{s} y_{0}\right|_{i=0} ^{j}-\mu, \\
j=1,2, \ldots, j_{0} .
\end{gathered}
$$

At $x=l$, we assume

$$
\begin{gathered}
\left.-\left.0.5 h y_{\bar{t}}\right|_{i=N} ^{j}=\left.a_{1}(z)(\tilde{y} y)_{\bar{x}}\right|_{i=N} ^{j}+a_{2}(z, y, \tilde{y}) y_{\bar{x}}\right)\left.\right|_{i=N} ^{j}, \\
j=1,2, \ldots, j_{0} .
\end{gathered}
$$

At $t=0$ we assume

$$
y\left(x_{i}, 0\right)=C_{0}\left(x_{i}\right), i=0,1, \ldots, N .
$$

Equation (2) for the defects is also approximated on mesh $\omega_{h \tau}$ using finite-difference schemes of the second and first kinds over space and time, respectively:

$$
\begin{gathered}
\left.\tilde{y}_{\bar{t}}\right|_{i} ^{j}=\left.\left(\left(a_{3}(z) \tilde{y}_{\bar{x}}\right)_{x}-(v \tilde{y})_{x}^{\circ}-\frac{1+k C^{c}}{l_{i}^{2}} \tilde{y}+\frac{d(\chi)}{l_{i}^{2}}\right)\right|_{i} ^{j}, \\
i=1,2, \ldots, N-1, j=1,2, \ldots, j_{0} .
\end{gathered}
$$

where $a_{3}(z)=0.5\left(d\left(z_{i}\right)+d\left(z_{i-1}\right)\right)$.

Condition $\tilde{y}_{N}^{j}=1, j=1,2, \ldots, j_{0}$ holds at $x=l$. The finite difference condition for the surface is given by

$$
\begin{gathered}
0.5 h\left(\left.\tilde{y}_{\bar{t}}\right|_{i=0} ^{j}+\left.(v \tilde{y})\right|_{i=1} ^{j}+\left.\frac{\left(1+k C^{c}\right) \tilde{y}}{l_{i}^{2}}\right|_{i=0} ^{j}-\left.\frac{d\left(z_{0}^{j}\right)}{l_{i}^{2}}\right|_{i=0} ^{j}\right) \\
=\left.a_{3}(z) \tilde{y}_{\bar{x}}\right|_{i=1} ^{j}-\left.\beta \tilde{y}\right|_{i=0} ^{j}-\gamma, \\
j=1,2, \ldots, j_{0} .
\end{gathered}
$$

The solution to the system of nonlinear difference equations is obtained iteratively.

\section{MODELING RESULTS}

To assess the model's applicability for calculating the diffusion redistribution of boron in silicon, we performed modeling for different initial conditions and parameters of the processes. The distribution profiles of boron in silicon, obtained by numerical calculations using our model, were compared with the corresponding experimental data from the literature. The results showed that our model allows us to obtain a reasonably good description of the boron distribution in silicon after low-energy implantation of boron ions and rapid thermal annealing, with allowance for the influence of implanted carbon on the diffusion of boron atoms.

Let us compare the results of modeling with the experimental data from [7]. The presented boron profiles were obtained at different stages of the following 


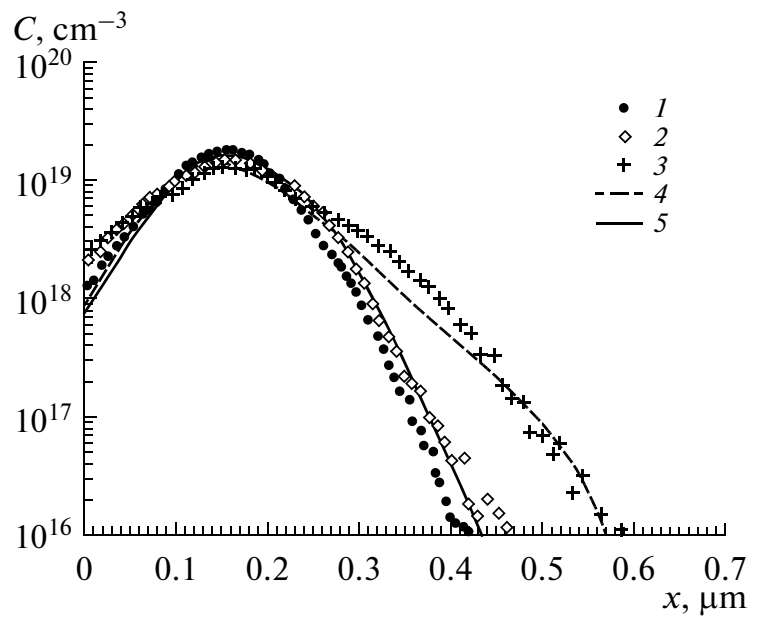

Fig. 1. Distributions of boron atoms implanted in silicon: (1) directly after implantation; (2) after thermal annealing of plates preliminarily implanted by carbon; (3) after thermal annealing of plates not implanted by carbon; (4) modeling the diffusion process with no allowance for the capture of interstitial silicon atoms by carbon atoms; (5) modeling the diffusion process with allowance for carbon.

process. Ion implantation was performed at an angle of $7^{\circ}$ in $\mathrm{Si}(100)$ substrates. Some of the substrates were first implanted with carbon at a dose of $5 \times 10^{14} \mathrm{~cm}^{-2}$ and an energy of $85 \mathrm{keV}$. Boron was then implanted in all of the substrates (at a dose of $2.5 \times 10^{14} \mathrm{~cm}^{-2}$ and an energy of $40 \mathrm{keV}$ ). Finally, the samples were subjected to rapid annealing at a temperature of $900^{\circ} \mathrm{C}$ for $20 \mathrm{~s}$. The profiles of boron distribution were measured at each stage of the process by means of second-ion mass spectrometry.

Figures 1 and 2 show the experimental results corresponding to boron and carbon implantation in silicon wafers, along with the results of calculations using our model and Silvaco's ATHENA software. Figure 1 presents the boron distributions in silicon at different stages of the aforementioned process. We can see that the proposed model adequately explains boron diffusion upon rapid thermal annealing both with and without carbon implantation.

For comparison, Fig. 2 shows the data obtained using Silvaco's ATHENA software, version 5.8.4.R (the CNET model of diffusion [8]). We can see that the model developed in this work gives a better estimate of the boron distribution profile in the depth of the substrate (at a depth of more than $0.3 \mu \mathrm{m}$ in this case) than the ATHENA software.

\section{CONCLUSIONS}

We have developed a physicomathematical model and computer software for numerically modeling the rapid thermal annealing of silicon structures implanted by boron and carbon ions. The migration of impurity atoms is examined in the form of diffusing

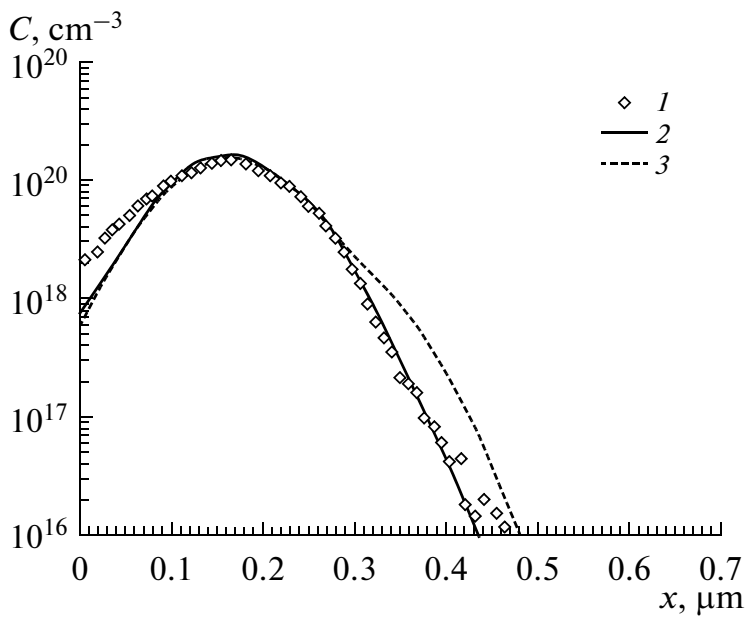

Fig. 2. Comparison of the results from modeling rapid boron thermal annealing in the presence of carbon: (1) the data obtained using SIMS; (2) the results from our own modeling; (3) the results from modeling using the ATHENA software.

"impurity atom-self-interstitial atom" complexes. The impact of an electric field, internal elastic stress, the bonding of interstitial silicon atoms with the carbon atoms, and the clustering of boron atoms on the diffusion of implanted impurities were examined and allowed for.

\section{ACKNOWLEDGMENTS}

This work was supported in part by the Belarusian Republican Foundation for Fundamental Research, project no. T11-159.

\section{REFERENCES}

1. Vanderpool, A. and Taylor, M., Nucl. Instrum. Methods B, 2005, vol. 237, p. 142 .

2. Komarov, F.F., Velichko, O.I., Dobrushkin, V.A., et al., Phys. Rev., 2006, vol. 74, no. 3, p. 035205.

3. Komarov, F.F., Mironov, A.M., Zayats, G.M., et al., Vacuum, 2007, vol. 8, p. 1184.

4. Fedotov, A.K., Velichko, O.I., and Dobrushkin, V.A., J. Alloys Comp., 2004, vol. 382, p. 283.

5. Pezoldt, J., Rybin, P.V., Kulikov, D.V., et al., Nucl. Instrum. Methods. B, 2000, vol. 166/167, p. 758.

6. Samarskii, A.A., Teoriya raznostnykh skhem (Theory of Difference Schemes), Moscow; Nauka, 1977.

7. Cowern, N.E.B., Cacciato, A., Custer, J.S., et al., Appl. Phys. Lett., 1996, vol. 68, no. 8, p. 1150.

8. Nelaev, V.V. and Stempitsii, V.R., Osnovy SAPR v mikroelektronike. Fizicheskoe modelirovanie tekhnologii $i$ pribora: uchebnoe posobie (Foundations of ComputerAided Design Systems in Microelectronics. Physical Simulation of Technologies and Devices: Student's Book), Minsk: BSUIR, 2006. 